\title{
JALONS POUR UNE RECHERCHE INTERDISCIPLINAIRE EN SANTÉ ET EN SCIENCES HUMAINES ET SOCIALES?: APPORTS D'UN SÉMINAIRE DE RECHERCHE
}

\author{
Joëlle Kivits et al.
}

S.F.S.P. | Santé Publique

2013/5 - Vol. 25
pages 579 à 586

ISSN 0995-3914

Article disponible en ligne à l'adresse:

http://www.cairn.info/revue-sante-publique-2013-5-page-579.htm

Pour citer cet article :

Kivits Joëlle et al., « Jalons pour une recherche interdisciplinaire en santé et en sciences humaines et sociales?: apports d'un séminaire de recherche »,

Santé Publique, 2013/5 Vol. 25, p. 579-586.

Distribution électronique Cairn.info pour S.F.S.P..

(c) S.F.S.P.. Tous droits réservés pour tous pays.

La reproduction ou représentation de cet article, notamment par photocopie, n'est autorisée que dans les limites des conditions générales d'utilisation du site ou, le cas échéant, des conditions générales de la licence souscrite par votre établissement. Toute autre reproduction ou représentation, en tout ou partie, sous quelque forme et de quelque manière que ce soit, est interdite sauf accord préalable et écrit de l'éditeur, en dehors des cas prévus par la législation en vigueur en France. II est précisé que son stockage dans une base de données est également interdit. 


\title{
Jalons pour une recherche interdisciplinaire en santé et en sciences humaines et sociales : apports d'un séminaire de recherche
}

\section{Benchmarks for interdisciplinary health and social sciences research: contributions of a research seminar}

\author{
Joëlle Kivits ${ }^{1}$, Cécile Fournier ${ }^{2}$, Jean-Christophe Mino ${ }^{3}$, Marie-Odile Frattini ${ }^{3}$, Myriam Winance ${ }^{2}$,
} Magali Robelet ${ }^{5}$, Céline Lefève ${ }^{4}$

\begin{abstract}
$\lceil\rightarrow$ Résumé
Cet article propose un retour réflexif sur une expérience de séminaire interdisciplinaire, à l'initiative de chercheurs en philosophie et en sociologie, et de médecins chercheurs en santé publique. L'objectif du séminaire était de s'interroger sur les mécanismes à l'œuvre lors de la mise en place et de la réalisation de recherches interdisciplinaires. Il s'agissait d'explorer les modalités concrètes d'articulation entre des recherches en santé et des recherches en sciences humaines et sociales, afin de mieux cerner les conditions, les tensions et les apports d'une telle rencontre et d'un travail commun. Ces questions étaient abordées à partir de l'analyse détaillée de cas de recherches récentes ou en cours. Quatre cas ont ainsi été étudiés. L'analyse des cas a fait émerger quatre questions « exemplaires », d'ordre épistémologique ou méthodologique, auxquelles les chercheurs qui mènent des travaux articulant santé et sciences humaines et sociales sont confrontés : les conditions institutionnelles et leurs effets sur la recherche ; la déconstruction de l'objet; l'engagement du chercheur sur son terrain ; l'articulation des méthodes de recherche. Trois préalables à la rencontre entre la recherche en sciences humaines et sociales et en santé ont été identifiés : l'interrogation mutuelle des postures et des espaces investis ; la prise de conscience des tensions liées aux positions institutionnelles et aux appartenances disciplinaires ; l'élaboration en commun et le va-et-vient entre différents types de savoirs pour que l'interdisciplinarité accompagne tout le processus de recherche.
\end{abstract}

Mots-clés : Interdisciplinarité ; Recherche ; Sciences sociales ; Santé publique.

\begin{abstract}
$[$ Summary
This article proposes a reflection on an interdisciplinary seminar, initiated by philosophy and sociology researchers and public health professionals. The objective of this seminar was to explore the mechanisms involved in setting up and conducting interdisciplinary research, by investigating the practical modalities of articulating health and human and social sciences research in order to more clearly understand the conditions, tensions and contributions of collaborative research. These questions were discussed on the basis of detailed analysis of four recent or current research projects. Case studies identified four typical epistemological or methodological issues faced by researchers in the fields of health and human and social sciences: institutional conditions and their effects on research; deconstruction of the object; the researcher's commitment in his/her field; the articulation of research methods. Three prerequisites for interdisciplinary research in social and human sciences and in health were identified: mutual questioning of research positions and fields of study; awareness of the tensions related to institutional positions and disciplinary affiliation; joint elaboration and exchanges between various types of knowledge to ensure an interdisciplinary approach throughout all of the research process.
\end{abstract}

Keywords : Interdisciplinarity; Research; Social sciences; Public health.

\footnotetext{
1 Université de Lorraine - APEMAC EA 4360 - 9 avenue de la Forêt de Haye - BP 184 - 54505 Vandœuvre-lès-Nancy.

2 INSERM U 988 - CNRS UMR 8211 - EHESS - CERMES 3 - Université Paris V.

3 Centre national de ressources sur les soins palliatifs.

4 Université Paris Diderot - SPHERE UMR 7219 - Centre Georges Canguilhem.

Université Lyon 2 - Centre Max Weber.
} 


\section{Introduction : un contexte favorable à la mobilisation des SHS dans la recherche dans le champ de la santé}

La recherche dans le champ de la santé est aujourd'hui marquée, en France comme à l'étranger, par la multiplication de travaux à caractère interdisciplinaire, se situant aux frontières entre disciplines de recherche médicales, épidémiologiques, de sciences humaines, sociales, économiques... Les travaux de ce type se développent de manière éparse, sans lien nécessaire les uns avec les autres, souvent avec un faible soutien institutionnel et peu d'encouragements financiers [1]. Ces recherches sont cependant porteuses de grandes potentialités, notamment dans le champ de la santé publique. Avec pour objet la connaissance et la surveillance de l'état de santé de la population, la compréhension de ses déterminants et l'identification d'interventions visant à améliorer la santé des populations et la réduction des inégalités face à la santé, la santé publique repose sur plusieurs disciplines. Deux domaines en santé publique témoignent particulièrement de la richesse des contributions interdisciplinaires. Celui de la prévention s'appuie sur une tentative d'appréhension globale des déterminants de la santé et des contextes favorables à la santé, impliquant des regards médicaux, sociaux, psychologiques mais également économiques et éthiques [2,3]. La problématique des inégalités sociales de santé fait également appel aux disciplines des sciences humaines et sociales (SHS) pour approfondir la compréhension des processus et guider l'action sur les causes [4-6].

Ces développements sont rendus favorables par l'existence d'intérêts réciproques. D'une part, l'intérêt des soignants et de l'administration de la santé va croissant pour les apports des SHS ; en témoignent leur place naissante dans les formations initiales et leur intégration dans les appels à projets de recherche. D'autre part, il existe un engagement accru de chercheurs en SHS dans une transmission de ces apports, pour éclairer et parfois accompagner les transformations en cours dans les modèles de soin [7]. Par ailleurs, s'exprime le besoin, pour les chercheurs engagés dans des recherches interdisciplinaires innovantes, de mettre en commun les approches, d'échanger sur les difficultés rencontrées et les solutions inventées. Ils doivent alors procéder à une «double traduction ${ }^{1}{ }^{\text {pour }}$

\footnotetext{
${ }^{1}$ Il existe une longue histoire d'essai de mobilisation des sociologues par l'État, visant à effectuer ce que Amiot appelait une « double traduction » : traduction des questions que l'administration se pose sur son environnement en thèmes sociologiques [...] et traduction des projets des chercheurs en thèmes opérationnels » (Amiot, 1986, cité par Herzlich et Pierret, 2010).
}

construire ensemble des projets à l'interface entre le monde de la santé et celui des sciences humaines et sociales [8].

Si le contexte est aujourd'hui favorable à la mobilisation des SHS, il serait cependant erroné d'interpréter comme nouvelle la rencontre entre le monde biomédical et les SHS. Cette rencontre parfois délicate dans les travaux menés en commun, s'ancre dans l'histoire de la constitution des disciplines impliquées. Comme le montrent Herzlich et Pierret [9], le développement d'une sociologie de la santé autonome en tant que secteur spécifique de la sociologie résulte d'un processus caractérisé par des approches pluridisciplinaires. Par ailleurs, l'évolution des problèmes et des pratiques de santé réactualise constamment la nécessité de cette rencontre. Au début des années 80 , l'épidémie du sida venait ainsi bousculer le modèle biomédical, amené à « s'ouvrir » à d'autres approches disciplinaires, dont celles des SHS $[10,11]$.

Confortée par les expériences et pratiques des chercheurs, quelles que soient leurs disciplines d'origine, et légitimée par son ancrage historique, la mobilisation des SHS dans la recherche dans le champ de la santé s'impose désormais. On connaît cependant mal les manières de faire et comment se traduit concrètement cette mobilisation sur le terrain. Ainsi s'est déroulé en 2008 et 2009 un séminaire de recherche méthodologique interdisciplinaire, initié par des chercheurs et professionnels en sciences humaines et sociales et en santé publique. L'objectif était d'explorer les conditions d'une rencontre entre disciplines de recherche issues des mondes médical et des sciences humaines et sociales ${ }^{2}$. Avec une visée pratique et à partir de recherches articulant santé et sciences humaines et sociales, il s'agissait d'identifier des points de passage obligés permettant à l'interdisciplinarité d'être intégrée tout au long du processus. Cet article témoigne de cette démarche et propose un retour réflexif sur cette expérience ${ }^{3}$.

\section{Séminaire méthodologique : organisation, principe de fonctionnement, réalisations}

Le séminaire a été initié par deux personnes: Céline Lefève, maître de conférences en philosophie de la

\footnotetext{
${ }^{2}$ http://www.rehseis.cnrs.fr/spip.php?article613\&lang=en.

${ }^{3}$ Le séminaire a été l'objet d'une communication lors d'un séminaire organisé par l'association "Sciences humaines \& santé», le 15 septembre 2012, sur le thème «SHS - Échanges de savoir : recherche, formation, action ». Les actes du colloque seront prochainement mis en ligne sur le site de l'association.
} 
médecine à l'Université Paris Diderot ${ }^{4}$ et Jean-Christophe Mino, médecin spécialiste de santé publique et chercheur, à l'époque praticien hospitalier à l'hôpital de la PitiéSalpêtrière et au Centre national de ressources sur les soins palliatifs ${ }^{5}$. Cinq personnes ont été associées à la démarche, choisies pour leur positionnement à l'interface entre les champs de la santé publique et des SHS (principalement la sociologie), avec, pour la plupart d'entre elles, des compétences doubles ${ }^{6}$.

Des choix préalables au séminaire ont été posés selon deux dimensions, celle de la problématique et celle de l'organisation matérielle. Premièrement, le regard porté à la recherche dans le champ de la santé s'est focalisé sur un aspect de celle-ci, à savoir les « services de santé »; ce choix répondait à l'intérêt des membres du séminaire pour les aspects organisationnels de la santé (au sens d'organisation sociale telle que l'entendent les sciences humaines et sociales), incluant aussi bien les dimensions d'organisation institutionnelle et les approches collectives, que la pratique clinique et les relations soignants-soignés. La réflexion s'inscrivait dans une visée compréhensive des processus en jeu dans les relations de soin, afin de ne pas se limiter à une acception étroite de la recherche clinique qui serait au seul service de l'amélioration des pratiques soignantes ou des résultats sanitaires, et donc principalement sous-tendue par des objectifs biomédicaux.

Deuxièmement, le parti-pris méthodologique était de ne pas s'interroger de manière abstraite ni normative sur ce que «devrait être » une collaboration interdisciplinaire entre SHS et santé publique, mais au contraire d'aborder ces questions à partir de l'analyse détaillée de cas de recherches récentes ou en cours, dont les critères de sélection étaient les suivants : recherches portant sur des sujets émergents liés aux questions actuelles du champ de la santé publique, se situant à l'articulation du champ de la santé publique et de celui des sciences sociales, ayant un objectif explicite d'aide à la compréhension des organisations et des services de santé, des pratiques professionnelles et des

\footnotetext{
${ }^{4}$ SPHERE UMR 7219 et Centre Georges Canguilhem.

${ }^{5}$ En collaboration avec le laboratoire SPHERE UMR 7219, le Centre Georges Canguilhem et en association avec la Société Française de Santé Publique.

${ }^{6}$ Marie-Odile Frattini, médecin chercheur au Centre national de ressources sur les soins palliatifs, doctorante en sciences sociales sur le handicap à l'EHESS ; Magali Robelet, maître de conférence en sociologie de la médecine et de la santé à l'Université Lyon 3/GRAPHOS ; Myriam Winance, sociologue du handicap à l'Inserm (Cermes3) ; Joëlle Kivits, sociologue, alors responsable de projets à la Société française de santé publique (SFSP) et enseignante à l'École de Santé Publique de Nancy; et Cécile Fournier, alors chargée de recherches à l'Institut national de prévention et d'éducation pour la santé (INPES), titulaire d'un DEA de sociologie à l’EHESS.
}

situations de soins (et parfois un objectif explicite de recherche appliquée et d'action).

Quatre cas ont ainsi été étudiés (Encadré 1), objets de réflexion pour une montée en généralité. L'ambition était d'identifier des dimensions qui posent régulièrement problème et que les chercheurs résolvent de manières diverses. Pour chaque cas de recherche, deux séances ont eu lieu. Une première séance était consacrée à la présentation d'un projet de recherche par un ou plusieurs chercheurs et à sa discussion, avec l'aide de deux discutants extérieurs. Lors d'une seconde séance, une mise en perspective était réalisée par les chercheurs permanents du séminaire, avec les promoteurs des recherches, pour discuter des aspects épistémologiques et méthodologiques de leurs travaux, et pour questionner plus particulièrement les conditions d'une fertilisation croisée entre les approches de santé publique et de SHS.

Un travail par phase a été réalisé, avec une première année consacrée à la recherche bibliographique et aux premières séances du séminaire, et une deuxième année consacrée à la poursuite du séminaire et à la recherche de financements pour valoriser les travaux et assurer leur poursuite.

\section{Les questions liées à l'articulation de recherches en santé publique et en SHS}

Les différentes recherches étudiées lors du séminaire ont permis d'identifier des questions " exemplaires », d'ordre épistémologique ou méthodologique, auxquelles les chercheurs qui mènent des travaux articulant santé publique et SHS sont confrontés. Quatre questions sont apparues de manière récurrente.

\section{Les conditions institutionnelles et leurs effets sur la recherche}

La question partagée par l'ensemble des chercheurs rencontrés, quelle que soit leur origine, est celle des conditions institutionnelles de la recherche. En amont du développement et de la réalisation de la recherche, elles renvoient à la « commande » initiant et encadrant l'activité de manière plus ou moins contraignante. Par commande, il est entendu tant les demandes externes que les demandes émanant de chercheurs et/ou de professionnels souhaitant développer un travail de recherche, avec, déjà à ce niveau, la possibilité d'une élaboration interdisciplinaire. Ces 
Encadré 1 : Cas présentés et discutés lors du séminaire méthodologique

Cas 1 : " L'expérience et la trajectoire des enfants et adolescents greffés "

- Chercheuse invitée: Isabelle Aujoulat, docteur en sciences de la santé publique (PhD).

- Discutants : Franck Guichet, sociologue, CSI, ENSMP et Philippe Barrier, philosophe et docteur en sciences de l'éducation à Paris XIII.

- Objet de la recherche : Étude qualitative des facteurs de non adhésion sur lesquels il serait possible d'agir par la relation de soin, avec pour finalité seconde d'aider les professionnels à développer une démarche systématique d'éducation auprès des patients et de leurs parents.

Cas 2 : "Démarche palliative dans la prise en charge aiguë des accidents vasculaires cérébraux graves. Étude multicentrique observationnelle prospective évaluant les décisions de limitations de traitements dans les unités neurovasculaires françaises " (Étude nationale TELOS/PHRC) ${ }^{2}$

- Chercheurs invités : Sophie Crozier, médecin neurovasculaire, docteur en éthique médicale, La Pitié-Salpétrière et Jean-Christophe Mino, médecin spécialiste de santé publique, titulaire d'une Habilitation à Diriger les Recherches (HDR), d'un doctorat en sciences de la santé publique (PhD) et d'un DEA «sciences sociales ».

- Discutants: Elsa Gisquet, alors sociologue à l'INCA et chercheure associée au CSO, et Florence Douguet, maître de conférences en sociologie à l'Université de Bretagne Sud, Lorient.

- Objet de la recherche : Étude des décisions de limitation et d'arrêt de traitement lors des accidents vasculaires cérébraux (AVC) graves, et de la démarche palliative mise en place, comprenant un premier volet quantitatif (enquête prospective par questionnaire avec recueil de données lors des AVC graves dans une vingtaine d'unités neurovasculaires) suivi d'un volet qualitatif (observation et entretiens dans quatre services prenant en charge des accidents vasculaires cérébraux).

Cas 3 : "Organisation, pratiques de soin, place des usagers et de l'entourage dans les services d'accompagnement des adultes souffrant d'un handicap psychique ${ }^{3}$

- Chercheurs invités : Carole Peintre, économiste de la santé au CREAI Île-de-France et Jean-Yves Barreyre, sociologue au CREAI Île-de-France.

- Discutants : Livia Velpry, maître de conférences en sociologie à l'Université Paris 8 et Fabrice Gzil, philosophe alors à l'Université Paris Diderot.

- Objet de la recherche: Méthodologie construite à partir des différentes recherches de chercheurs invités : recherches appliquées, co-construites par des chercheurs, des professionnels, des patients et leur entourage.

Cas 4 : "Épidémiologie et anthropologie sociale : intérêts et difficultés d'une rencontre. L'exemple de l'unité d'épidémiologie des virus oncogènes de l'Institut Pasteur $n^{4}$

- Chercheur invité: Christophe Perrey, pharmacien et anthropologue à l'Unité de recherche clinique Robert Debré et à l'Unité de recherche IGR.

- Discutant: Guillaume Lachenal, maître de conférences en histoire de la médecine, Université Paris Diderot/Laboratoire SPHERE UMR 7219

- Objet de la recherche : Présentation de deux recherches menées de front, « sur » et « avec » une unité d'épidémiologie : une ethnographie de l'unité d'une part, et une étude des dynamiques spatiotemporelles des maladies associées aux rétrovirus d'autre part (étude du lien avec des migrations humaines).

${ }^{1}$ Aujoulat I, Deccache A, Charles AS, Janssen M, Struyf C, Pélicand J, Ciccarelli O, Dobbels F, Reding R. Non-adherence in adolescent transplant recipients: The role of uncertainty in health care providers. Pediatric Transplantation 2011; 15(2): 148-56.

Aujoulat I, Reding R. L’adolescent greffé hépatique : Quels défis spécifiques lors de la transition vers une gestion autonome de sa santé ? Percentile $2012 ; 17(4): 24-9$.

${ }^{2}$ Mino JC. Soins Intensifs. La technique et l'humain, PUF, Paris, 2012.

Crozier S. «Le pari éthique de la complexité. Action médicale dans le champ des AVC graves», thèse d'Éthique, Université Paris Sud, 2012.

${ }^{3}$ Barreyre JY, Peintre C. Évaluer les besoins des personnes en action sociale - Enjeux, méthodologie, outils. Paris, Dunod, 2004. Barreyre JY,

Bouquet C, Fiacre P, Peintre C. Les trajectoires institutionnelles et scolaires des enfants passées en CDES. Études et résultats 2007, n 580, 8 p.

${ }^{4}$ Perrey C, de Thé G. Le souple et le dur : les sciences humaines au secours des sciences biomédicales. Paris, CNRS, 2009.

Perrey C. Un ethnologue chez les chasseurs de virus. Enquête en Guyane française. L'Harmattan, Collection Logiques sociales, 2012 
cadres peuvent être temporels, financiers mais également épistémologiques et méthodologiques, voire d'obligation de résultats dans le contexte de recherche appliquée. Est alors posée la question de l'autonomie du chercheur: comment la garantir tout en restant attentif aux questions des acteurs de terrain et/ou des commanditaires?

L'analyse des cas a permis de caractériser les conditions dans lesquelles se déroule la recherche, de comprendre la façon dont elles peuvent influencer le cours de la recherche (ses modalités, son objet, son contenu) et d'en tirer les conséquences pour en préserver la pertinence et la rigueur. Ainsi, si l'on prend l'exemple des recherches analysées, dans le cas 1 , le chercheur a pu «négocier» la commande; dans le cas 2, les chercheurs ont obtenu un financement pour un projet plutôt quantitatif et biomédical, mais ont pu conserver une grande autonomie dans les choix scientifiques, dans la limite du budget et du temps impartis ; dans le cas 3 , une recherche de financements complémentaires a été effectuée afin de disposer de plus d'autonomie dans la mise en œuvre d'une activité de recherche répondant à une commande; enfin dans le cas 4, le chercheur s'est trouvé dans une situation initiale d'autonomie complète, mais qui rendait difficile la réalisation de son terrain du fait du peu d'interactions avec l'équipe dans laquelle il était accueilli.

Entre « autonomie totale » et « être tenu complètement », le chercheur est ainsi placé dans un large spectre de « positions » possibles, qui dépendent du type de demande, des délais, des compétences et approches mobilisées, des commanditaires, de l'utilisation prévue des résultats et du degré de liberté ou de maîtrise que le chercheur peut avoir vis-à-vis de ces différents aspects. Parce que cette position influence la façon de conduire la recherche et les résultats qu'elle peut produire, il importe à la fois d'en tenir compte et d'en rendre compte.

\section{Comment opérer la " déconstruction " de l'objet dans une recherche dans le champ de la santé ?}

Au départ, il s'agissait d'identifier comment les chercheurs rencontrés « construisaient » un objet de recherche. Étape fondamentale de la démarche de recherche, quelle que soit la discipline, la déconstruction de l'objet de recherche est un enjeu majeur en SHS $[12,13]$ : elle consiste tout d'abord à "déconstruire " l'objet ou à opérer une «rupture épistémologique », c'est-à-dire à identifier les opinions communes sur l'objet pour s'en distancier, puis à définir l'objet dans une perspective de recherche, avec l'élaboration d'une problématique. Cette étape est plus complexe dans des recherches dites appliquées et appelle d'emblée une négociation entre regards disciplinaires. Au cours du séminaire, ont donc été discutés les déplacements effectués, les médiations éventuelles avec les demandeurs, les effets de ces déplacements sur la production de connaissance et sur l'utilisation possible de cette connaissance.

En particulier, la plupart des cas étudiés concernaient des recherches parties d'une question qui se posait aux acteurs de terrain, ou d'un problème rencontré par ces acteurs. Il s'agissait souvent au début de questions floues, de situations que les acteurs ne savaient pas bien définir et saisir. Du point de vue de la démarche de recherche, il y avait souvent un objectif de compréhension d'une question complexe, qui devait permettre dans un second temps d'agir sur la réalité sociale.

Ainsi dans le premier cas analysé, la recherche a commencé par une revue de littérature biomédicale qui orientait fortement l'étude; il a fallu que la chercheuse pointe les manques dans cette revue de littérature pour justifier le recours à une littérature de sciences sociales et la mise en place d'une approche inductive. Dans l'exemple du cas 2 , une première séquence d'observations sur le terrain et un changement de calendrier ont mené à une redéfinition de l'objet: la question initiale sur les limitations et arrêts de traitement s'est élargie à celle des stratégies de prise en charge des AVC graves. Cette étape de dé/ construction de l'objet s'est faite en lien étroit avec les acteurs impliqués dans le troisième cas analysé, par la mise en place d'un comité de pilotage permettant une construction partagée de l'objet.

La recherche dans le champ de la santé s'inscrivant souvent dans une perspective de "commande » médicale ou d'institution du système de santé, l'enjeu est de pouvoir rendre collaborative cette phase de dé/construction de l'objet. L'analyse des cas montre en effet que l'implication de l'ensemble des acteurs concernés est centrale, à un double niveau : celui du dialogue entre disciplines et celui du dialogue entre chercheurs et acteurs.

\section{L'engagement du chercheur sur son terrain et le lien entre recherche et intervention}

La question classique de l'engagement du chercheur sur son terrain $[14,15]$ a été reformulée du fait de la spécificité des recherches présentées. Est apparue dans le courant des discussions une manière particulière pour les chercheurs d'arriver à intéresser les différents partenaires de la recherche. Dès lors, la position n'est plus celle, asymétrique, du chercheur qui s'engage vis-à-vis des personnes 
concernées, mais les positions deviennent plus symétriques, avec la coexistence d'intérêts réciproques pour la recherche. Ici s'articulent questions épistémologiques, éthiques et politiques, à explorer plus avant [16].

Les particularités de la santé publique et cette manière d'envisager la collaboration modifientégalement le rapport à l'action : l'intérêt vient du fait que l'on est d'emblée dans l'action, ou du moins que l'action ou les retombées de la recherche sontà l'horizon du travail. Comme Robert Strauss appelant à une "sociology with» [17] menée «avec » les acteurs, ont été discutées les conditions de possibilité d'une approche de SHS «avec » ou «aux côtés » des acteurs de santé publique.

Dans les exemples apportés par les quatre cas, cela se traduit dans le premier cas par des retours réguliers des résultats de la recherche au comité scientifique, et par la co-construction avec l'équipe soignante d'une offre de prise en charge incluse dans la recherche et définie en fonction de ses résultats. Dans le deuxième cas, le travail « avec » les soignants se fait au sein de l'équipe de recherche même, le rapport entre recherche et action étant particulièrement étroit : certains membres de l'équipe «attendent» des résultats directement applicables, or le questionnement des chercheurs sur l'objet vient remettre en cause la demande initiale, qui est ainsi amenée à évoluer. Dans le troisième cas étudié, le rapport à l'action est fort puisque la recherche résulte d'une commande, cependant la réflexion dépasse la seule aide à la décision. Enfin dans le quatrième cas, le chercheur qui se trouve dans une situation très autonome, libre de définir son propre objet de recherche, fait face à la difficulté d' « intéresser » l'équipe qu'il prend pour objet; il ne parvient à développer un travail «avec » l'équipe qu'à partir du moment où il s'engage avec ses membres dans un projet de recherche parallèle.

Les cas étudiés illustrent ce que défend le Réseau des jeunes chercheurs santé et société ${ }^{7}$ : une pratique de recherche non pas «appliquée » mais «impliquée», c'està-dire assumée comme une activité sociale et collaborative, avec le souci de «dialoguer sur ce que sont souvent des questions communes, approchées différemment » et de « concevoir des formes de collaboration négociées, impliquées et - tant qu'à faire - rémunérées » [18]. Cette position est nécessairement liée à un engagement à la fois d'ordre scientifique et politique, qui nécessite un recul par rapport à la manière dont les objets sont définis et analysés par les divers acteurs impliqués.

${ }^{7}$ http://www.reseau-sante-societe.org/

\section{L'articulation des méthodes de recherche}

L'interdisciplinarité des recherches dans le champ de la santé appelle souvent des dispositifs méthodologiques mixtes, recourant tant aux méthodes quantitatives que qualitatives. Se pose alors la question de l'articulation des approches, l'approche quantitative étant généralement associée à la recherche épidémiologique ou clinique, tandis que l'approche qualitative relèverait de la recherche en SHS [19].

Cette articulation a été analysée dans ce contexte bien particulier où les conceptions médicales étaient souvent le point de départ des projets examinés, et discutée au-delà du seul cadre du choix méthodologique. En effet, cette articulation se fait en lien étroit avec la construction de l'objet. Par exemple, dans l'étude de cas 2, le questionnement de départ était médical et partait de l'expérience d'un service particulier sur les limitations et arrêts de traitement lors des AVC graves. L'étude devait commencer par un volet quantitatif national sur les pratiques, avec un questionnaire construit à partir des manières de penser et de faire de ce service, le volet qualitatif étant prévu dans un second temps pour mieux comprendre les résultats du volet quantitatif. En pratique, le déroulement du projet a inversé le calendrier et par conséquent la logique de recherche. Commencer par l'observation a permis de s'apercevoir que les questions ne se posaient pas de la même manière dans tous les services, que le recueil quantitatif de données risquait d'influer sur les pratiques et que la question initiale était trop étroite.

Certaines conditions ont été mises en évidence pour une articulation des méthodes et non une simple juxtaposition : le choix du dispositif de recherche, la position du chercheur sur son terrain, la place donnée à une démarche inductive permettant d'aborder de nouvelles dimensions de l'objet... Cette réflexion se nourrit des nombreuses publications récentes sur les méthodes mixtes de recherche, champ en plein développement, pour approfondir les questions qui se posent spécifiquement dans le champ de la santé publique [20, 21].

\section{Discussion}

L'organisation du séminaire a favorisé un dialogue constructif et un croisement des approches, à deux niveaux : d'une part, entre membres permanents du séminaire dans l'élaboration de l'objet du séminaire et de son déroulement 
qui a nécessité des temps de rencontre, le partage de références bibliographiques et l'élaboration d'un vocabulaire partagé ; et d'autre part entre membres permanents et membres invités, dans les moments de présentation des recherches et dans le travail d'analyse transversal réalisé après chaque présentation. Les travaux du séminaire contribuent ainsi à une meilleure compréhension des modalités concrètes d'articulation de ces approches de recherche.

À ce stade d'avancée des travaux, il est possible, à partir de l'analyse des cas, de dégager trois préalables à la rencontre entre la recherche en SHS et en santé publique.

Le premier préalable est celui du positionnement des chercheurs impliqués dans des recherches interdisciplinaires en santé, où le travail collectif interdisciplinaire en SHS se confronte à un travail avec des professionnels de santé. Comme le développent Marc Bessin et le Réseau des jeunes chercheurs santé et société, on se heurte aux « enjeux de la production de connaissance [...] dans ce domaine où l'implication individuelle et la dynamique collective posent de façon singulière le rapport du savant au politique » [22]. Avec ces auteurs, nous nous demandons « si le champ de la santé, comme d'autres (social, genre, discriminations...) tout aussi enclins à se confronter directement aux enjeux politiques, n'incite pas davantage à une posture qui ose interroger les espaces académiques et sociaux investis [c'est-à-dire repenser les oppositions structurantes du milieu scientifique (recherche collective/individuelle ; isolement/ dispersion; visée scientifique/professionnalisation; savant/politique)], sans renier la prétention à la rigueur des sciences sociales ».

Avoir conscience des tensions liées aux positions institutionnelles de chacun et aux appartenances disciplinaires constitue le deuxième préalable: une tension entre visée scientifique et professionnalisation que nous avons déjà soulignée ; mais aussi des positions plus ou moins fragiles dans le milieu de la recherche (chercheurs statutaires, chercheurs contractuels, chercheurs travaillant dans des associations professionnelles ou des agences de santé...) ; une évaluation sur des critères différents (la production d'articles scientifiques disciplinaires pour les chercheurs statutaires, et la production d'écrits de l'ordre de recommandations professionnelles pour les acteurs de santé publique) ; des logiques de publication différentes (valorisation des articles ou des ouvrages signés seuls en SHS, valorisation des articles collectifs en santé publique)...

Enfin, des traductions permanentes sont nécessaires [8], avec une ouverture réciproque des chercheurs issus de la santé publique et des sciences humaines et sociales, qui est facilitée par l'implication de chercheurs ayant des doubles formations. Il est indispensable de passer du temps à une élaboration en commun et à des va-et-vient entre différents types de savoirs pour que l'interdisciplinarité accompagne tout le processus de recherche. Les chercheurs positionnés aux points d'interface sont amenés à entrer en dialogue avec une multitude d'acteurs différents, ils doivent " comprendre et raisonner dans une pluralité de grammaires", ce qui les met en situation de "traducteur", «à l'interface entre les mondes intime, médical, social et politique » [18].

Ces préalables et la rencontre elle-même entre chercheurs en SHS et dans les autres disciplines de la santé publique ne pourront se réaliser sans un soutien institutionnel et financier : ce soutien est un élément crucial pour permettre - dans la durée - le rapprochement de chercheurs travaillant sur le même domaine mais dans des disciplines et des universités différentes et dans des laboratoires où ils sont souvent seuls à travailler sur le thème de la santé.

Aucun conflit d'intérêt déclaré

\section{Remerciements}

Ce séminaire interdisciplinaire a pu se dérouler grâce à la participation et la contribution des chercheurs ayant accepté de présenter leurs recherches, et grâce aux discutants. Nous tenons ainsi à remercier (par ordre alphabétique) : Isabelle Aujoulat, Jean-Yves Barreyre, Philippe Barrier, Sophie Crozier, Florence Douguet, Elsa Gisquet, Franck Guichet, Fabrice Gzil, Guillaume Lachenal, Carole Peintre, Christophe Perrey et Livia Velpry.

\section{Références}

1. Moatti JP, Spira A, Singh Manoux A, Thiebaut R. Reconnaître la spécificité de la recherche en santé publique pour améliorer son impact scientifique, sanitaire et sociétal. Santé publique. 2012;24(5):383-5.

2. Inspection Générale des Affaires Sociales. Santé, pour une politique de prévention durable : rapport annuel 2003. La Documentation française ; 2003.

3. Potvin L. Yes! More research is needed; but not just any research. Int J Public Health. 2009;54:127-8.

4. Abel T, Frolich K L. Capitals and capabilities: linking structure and agency to reduce health inequalities. Social Science \& Medicine. 2012;74:236-44.

5. Goldberg M, Melchior M, Leclerc A, Lert F. Les déterminants sociaux de la santé : apports récents de l'épidémiologie sociale et des sciences sociales de la santé. Sciences sociales et santé. 2002;4:75-128. 
6. Ridde $V$, Guichard A. Réduire les inégalités sociales de santé : aporie, épistémologie et défis. In: Niewiadomski C, Aiach P (dir.). Lutter contre les inégalités sociales de santé. Politiques publiques et pratiques professionnelles. Presses de I'EHESP; 2008.

7. Lefève $C$, Mino JC. Former de vrais thérapeutes. La place des sciences humaines et sociales dans les études de médecine. Études. 2011; 4142:187-98.

8. Akrich $\mathrm{M}$, Callon M, Latour B. Sociologie de la traduction : textes fondateurs. Presses de l'École des Mines; 2006.

9. Herzlich C, Pierret J. Au croisement de plusieurs mondes : la constitution de la sociologie de la santé en France (1950-1985). Revue française de sociologie. 2010;51(1):121-48.

10. Deschamps JP. Les sciences humaines, victimes de la trithérapie. Sante Publique. 2001;13(3): 295-9.

11. Calvez C. De la santé publique aux sciences sociales. Les recherches sur le sida en France entre 1985 et 1997. Socio-logos. Revue de l'association française de sociologie. 2012;7. http://sociologos.revues.org/2627.

12. Becker H. Les ficelles du métier. Comment conduire sa recherche en sciences sociales. La Découverte; 2002.

13. Quivy R, Van Campenhoudt L. Manuel de recherche en sciences sociales. Dunod ; 2011.

14. Fassin D. L'anthropologie entre engagement et distanciation. Essai de sociologie des recherches en sciences sociales sur le sida en
Afrique. In : Becker C, Dozon JP, Obbo C, Touré M (dir.). Vivre et penser le sida en Afrique. IRD/Karthala/Codesria; 1998.

15. Herreros G. Pour une sociologie d'intervention. Eres; 2009.

16. Chabrol F, Girard G. Se confronter au terrain. Expériences et postures de recherche. Éditions ANRS; 2010.

17. Straus R. Medical sociology: a personal fifty year perspective. Journal of health and social behavior. 1999;40:103-10.

18. Chabrol F, Hunsmann M, Kehr J. Réaliser un doctorat en sciences sociales de la santé : financements, pratiques de recherche et enjeux de professionnalisation. Socio-logos. Revue de l'association française de sociologie. 2012;7:http://socio-logos.revues.org/2629.

19. Teddlie C, Tashakkori A. Foundations of Mixed Methods Research: Integrating Quantitative and Qualitative Approaches in the Social and Behavioral Sciences. Sage publications; 2008.

20. Jauffret-Roustide M. Sciences sociales et épidémiologie: des approches méthodologiques qui se complètent, à propos de la question des pratiques à risque chez les usagers de drogues. $\mathrm{BEH}$. 2006;2-3:21-3.

21. Guével MR, Pommier J. Recherche par les méthodes mixtes en santé publique : enjeux et illustration. Santé Publique. 2012;24(1):23-38.

22. Bessin M, Chabrol F, Kehr J, Michels D. Le " Réseau des jeunes chercheurs Santé et Société ": un acteur des sciences sociales de la santé. Socio-logos. Revue de l'association française de sociologie. 2012;7. http://socio-logos.revues.org/2671. 